\title{
Preventable trauma death rate in Daegu, South Korea
}

\section{Sungbae Moon', Suk Hee Lee', Hyun Wook Ryoo', Jong Kun Kim', Jae Yun Ahn', Sung Jin Kim², Jae Cheon Jeon², Kyung Woo Lee ${ }^{3}$, Ae Jin Sung ${ }^{3}$, Yun Jeong Kim ${ }^{4}$, Dae Ro Lee ${ }^{4}$, Byung Soo Do ${ }^{5}$, Sin Ryul Park ${ }^{5}$ Jin-Seok Lee ${ }^{6}$}

Department of Emergency Medicine, ${ }^{1}$ Kyungpook National University School of Medicine, Daegu; ${ }^{2}$ Keimyung University Dongsan Medical Center, Keimyung University School of Medicine, Daegu; ${ }^{3}$ Daegu Catholic University Medical Center, Daegu; ${ }^{4}$ Daegu Fatima Hospital, Daegu; ${ }^{5}$ Yeungnam University College of Medicine, Daegu; ${ }^{6}$ Department of Health Policy and Management, Seoul National University College of Medicine, Seoul, Korea

Objective This study investigated the preventable death rate in Daegu, South Korea, and assessed affecting factors and preventable factors in order to improve the treatment of regional trauma patients.

Methods All traumatic deaths between January 2012 and December 2012 in 5 hospitals in Daegu were analyzed by panel review, which were classified into preventable and non-preventable deaths. We determined the factors affecting trauma deaths and the preventable factors during trauma care.

Results There were overall 358 traumatic deaths during the study period. Two hundred thirty four patients were selected for the final analysis after excluding cases of death on arrival, delayed death, and unknown causes. The number of preventable death was 59 (25.2\%), which was significantly associated with mode of arrival, presence of head injury, date, and time of injury. A multivariate analysis revealed that preventable death was more likely when patients were secondly transferred from another hospital, visited hospital during non-office hour, and did not have head injuries. The panel discovered 145 preventable factors, which showed that majority of factors occurred in emergency departments (49.0\%), and were related with system process (76.6\%).

Conclusion The preventable trauma death rate in Daegu was high, and mostly process-related.

Keywords Death; Wounds and injuries; Outcome and process assessment; Secondary prevention

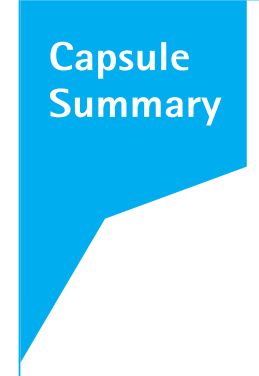

What is already known

All studies analyzing trauma deaths ultimately aim to reduce preventable death rate and improve the local trauma management system. The preventative death rate in South Korea varied from 30\% to 50\%.

What is new in the current study

The preventable death rate in Daegu was 25.2\%. This study assessed factors related to the preventable death rate to obtain baseline data to help organize the regional trauma care system.
Received: 31 July 2015

Revised: 15 October 2015

Accepted: 18 October 2015

Correspondence to: Suk Hee Lee Department of Emergency Medicine, Kyungpook National University School of Medicine, 680 Gukchaebosang-ro, Jung-gu, Daegu 41944, Korea E-mail:mycozzy@naver.com

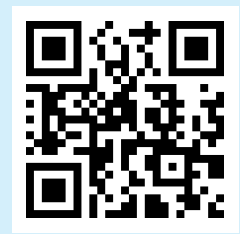

How to cite this article:

Moon S, Lee SH, Ryoo HW, Kim JK, Ahn JY, Kim SJ, Jeon JC, Lee KW, Sung AJ, Kim YJ, Lee DR, Do BS, Park SR, Lee JS. Preventable trauma death rate in Daegu, South Korea. Clin Exp Emerg Med 2015;2(4):236-243.

This is an Open Access article distributed under the terms of the Creative Commons Attribution Non-Commercial License (http:// creativecommons.org/licenses/by-nc/3.0/). 


\section{INTRODUCTION}

In 2012, trauma was the third leading cause of death in the Daegu area after neoplasm and cardiovascular disease. The majority (64\%) of trauma deaths took place in people with productive ages between 15 and 64. Deaths of people in this age group could lead to a socioeconomic burden in the community. Therefore, analyzing trauma management systems is necessary to decrease the number of preventable deaths from trauma.

The concept of preventable death was first proposed as a death that was partially or completely avoidable after effective treatment. ${ }^{2}$ Under a more modern definition of preventable death, death is considered preventable if the deceased is transferred to a hospital with the most adequate facility and faculty in an optimal time and if complete information about the clinical diagnosis and treatment is provided. Preventable death is defined by the following three criteria: 1) the patient survived the damage and impact from the early stage of trauma, 2) the resuscitation and treatment thereafter deviated from the advanced trauma life support guideline, and 3) errors during the treatment directly or indirectly contributed to the patient's death., ${ }^{3,4}$

Preventable death is expressed as the preventable death rate (PDR) mathematically. PDR is used as a tool for evaluating trauma management systems. Repeating evaluation of trauma management outcome using PDR will enable consistent quality control of trauma management systems and clarify which factors are preventable and related to trauma deaths.

PDR has been investigated thrice in South Korea; the rate was $50.4 \%$ in $1997-1998,39.6 \%$ in 2003-2004, and $32.6 \%$ in 20062007. ${ }^{5-7}$ PDR in developed countries, depending on the research and geographic region, varies from $1 \%$ to $71 \% .{ }^{8}$ All studies analyzing trauma deaths ultimately aim to reduce PDR and improve the local trauma management system, regardless of the current rate. However, PDR may vary by region, even in the same country, because the quality and quantity of prehospital care, the system for transferring patients between hospitals (i.e., interhospital transfer), and the number of emergency medical personnel may differ geographically. Therefore, the assessment of preventability should be based not on a single study but rather on periodical, serial, and identical studies. Such studies should be conducted after improving trauma management systems through data analysis.

In the previous studies from Korea, the number of hospitals, scope, and methods varied, making a detailed comparison impossible; few data are available that represent individual regions. This study investigated PDR in Daegu and assessed factors related to PDR to obtain baseline data to help organize the regional trauma care system in Daegu.

\section{METHODS}

\section{Study design and setting}

As of 2012, Daegu had a population of 2,527,566 and covered $883.63 \mathrm{~km}^{2}$ of land area. Daegu has 2,572,363 m of road, consisting of 5,268 $\mathrm{m}$ of unopened road and 2,567,095 $\mathrm{m}$ of completely paved road. ${ }^{9}$ The area has 26 medical facilities in operation, consisting of 1 regional emergency medical center, 4 local emergency medical centers, 8 local emergency medical facilities, and 13 nondesignated medical facilities. One regional emergency medical center and 4 local emergency medical centers participated in this study. The number of patients that visited the regional emergency medical center was 49,254 ; the number of patients that visited the 4 local emergency medical centers was 143,391; and the number of severe trauma patients that visited 5 emergency medical centers was $4,555 .{ }^{10}$ The regional emergency medical center participating in this study was selected as a specialized trauma care center in 2010, but the trauma center had not yet started operating. The 4 local emergency medical centers did not have any particular protocols. Prehospital emergency medical services (EMS) provided trauma care with standard protocols for EMS providers but did not have separate protocols for interhospital transfers.

This cross-sectional study included a retrospective review of the EMS medical records, transfer notes, progress notes, image readings, and death certificates of all patients. We did not review the previous hospital records of transferred patients. This study was approved by the institutional review board (2014-04-036), which waived informed consent.

\section{Study population}

The study included all trauma-related deaths among patients visiting the emergency department (ED) in the 5 emergency medical facilities from January 1, 2012, to December 31, 2012. We defined trauma death as cases in which trauma was the direct cause of death, with the exception of drowning, strangulation, and intoxication. Among all trauma deaths, patients who were dead on arrival, dead after $\geq 7$ hospital days, and dead due to unknown causes were excluded.

\section{Study protocol and data source}

The study protocol was performed using the following process. Board-certified emergency physicians from the 5 target hospitals, who did not directly participate in the patients' treatment processes, evaluated the preventability of trauma death as a preliminary investigation. The investigators consisted of 10 physicians, two of whom visited each target hospital to collect and review the data. We provided an educational session for the investiga- 
tors concerning the research methods before the study, and the investigations followed the protocol given at this session. The investigators reviewed the medical records and image readings of each patient, completed a form containing problem codes, and determined the preventability of deaths based on the presence of medically preventable factors. The 2 investigators did not interact during the evaluation. After the evaluation, we examined consistency between the findings of the 2 investigators. If the investigators' results did not match, the investigators went through an agreement process between them. In case the disagreement continued even after this primary process, a conclusion was made through meetings between the emergency physicians who took part in the investigation and an advisory panel of other specialists from the 5 target hospitals. Six specialists took part in this advisory panel-2 general surgeons, 2 thoracic surgeons, 1 neurosurgeon, and 1 trauma surgeon. The emergency physicians explained the preventability of their cases and drew unanimous conclusions after discussing the case with the panel.

\section{Main outcome}

The main outcome was PDR in Daegu. The case was defined as a preventable death if the 2 emergency physicians agreed or if the advisory panel unanimously agreed.

\section{Measurements and variables}

The study investigated the patients' age, sex, mechanism of injury, cause of death, place of death, day of injury, time from accident to arrival at $E D$, time from arrival at $E D$ to death, and mode of arrival. The severity of injury was determined using the Abbreviated Injury Scale and Injury Severity Score. In cases of unknown scores on the Glasgow Coma Scale, the alert/verbal/painful/unresponsive scale was converted to calculate the Trauma and Injury Severity Score as a means to evaluate the expected survival rate. ${ }^{11}$

Preventable factors during trauma care were divided into two categories: structure-related and process-related. Place of death was divided into the following categories: prehospital, interhospital transfer, ED, intensive care unit (ICU), operating room, and general ward. Structure-related preventable factors were defined as failures of the trauma system in cases that required adequate treatment within an optimal time. This definition included the inability to provide adequate emergency medical staff or facilities, thereby resulting in delays in patients' transfer, diagnosis, or treatment, and consequently demanding equipment or system revision. Process-related preventable factors included the following categories: inadequacy in treatment or management, inadequacy in diagnosis, inadequacy in technique, and others. A determination of inadequacy in treatment was based on the treatment process described in the Advanced Trauma Life Support algorithm. We separately analyzed problematic care as a cause of death according to when the preventable factor was developed, and then divided the cases into overall preventability and hospital preventability. Based on the problems with each process, death was defined as preventable $(P)$ if the chance of preventability was $25 \%$ or higher. If the chance was under $25 \%$, then death was defined as non-preventable (NP). Thus, the final PDR was defined as preventability of $25 \%$ or higher.

\section{Statistical analysis}

The clinical characteristics of patients are presented as the frequency and percentage, mean with standard deviation, or median with interquartile range, as appropriate. We tested comparisons of categorical and continuous data using Student's t-test and Pearson's chi-square test, respectively. To determine the factors affecting the preventability of trauma death, we performed multivariate logistic regression analysis. All variables used in univariate analysis were included in multivariate logistic regression analysis. The method of independent variables selection was backward elimination. The results of logistic regression analyses were presented as odds ratio (OR) and 95\% confidence interval (CI). Pvalues $<0.05$ were defined as statistically significant. The interinvestigator agreement in the initial preventability judgment was presented with Kappa values. Kappa values were evaluated for overall care and the in-hospital phase, respectively. Statistical analysis was conducted using IBM SPSS Statistics ver. 21.0 (IBM Corp., Armonk, NY, USA).

\section{RESULTS}

\section{General characteristics of study patients}

The total number of trauma deaths during the study period was 358. Among these cases, 54 patients were dead on arrival, and 6 deaths were of unknown cause owing to insufficient diagnostic examination after hospital arrival. We excluded 64 patients who died after $\geq 7$ hospital days, resulting in a final study population of 234 patients (Fig. 1). The mean patient age was 57.3 years, and 170 patients (72.6\%) were male. Motor vehicle accidents were the most common mechanism of death, head injury the most common cause, and ED the most common place. The mean Injury Severity Score was $24.8 \pm 13.8$. The median time from accident to arrival at ED was 1.00 hour, and the median time from arrival at ED to death was 2.74 hours (Table 1).

\section{Analysis of factors associated with PDR}

PDR in Daegu was 25.2\%. The differences between P and NP 


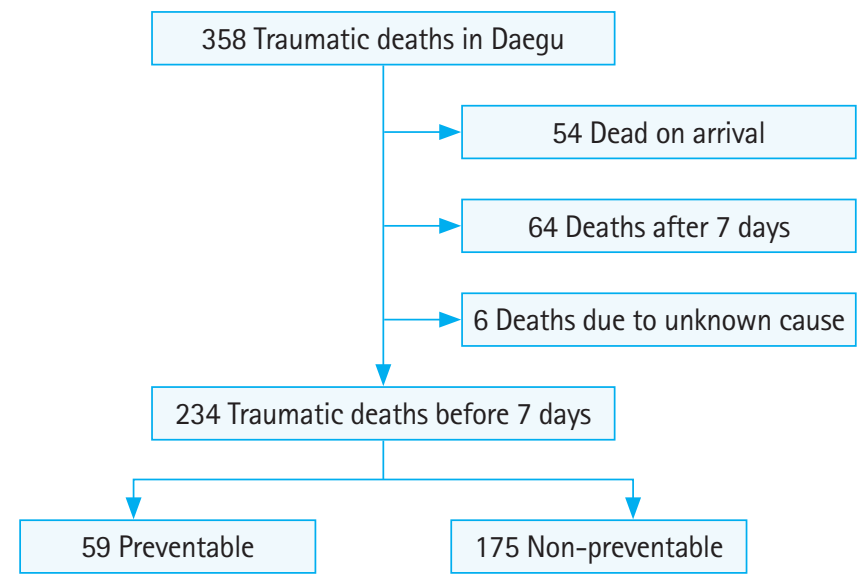

Fig. 1. Preventable death rate study flow diagram.

Table 1. General characteristics of study patients

\begin{tabular}{|c|c|}
\hline Variable & Value \\
\hline \multicolumn{2}{|l|}{ Sex } \\
\hline Male & $170(72.6)$ \\
\hline Female & $64(27.4)$ \\
\hline Age (yr) & $57.3 \pm 19.0$ \\
\hline \multicolumn{2}{|l|}{ Mechanism of injury } \\
\hline Motor vehicle accident & $159(67.9)$ \\
\hline Fall & $54(23.1)$ \\
\hline Blunt trauma & $5(2.1)$ \\
\hline Penetrating trauma & $1(0.4)$ \\
\hline Unknown & $14(6.0)$ \\
\hline Others & $1(0.4)$ \\
\hline \multicolumn{2}{|l|}{ Cause of death } \\
\hline Head injury & $118(50.4)$ \\
\hline Multiple injuries & $53(22.6)$ \\
\hline Hemorrhage & 39 (16.7) \\
\hline Organ failure/sepsis & $11(4.7)$ \\
\hline Preexisting disease & $2(0.9)$ \\
\hline Others & $11(4.7)$ \\
\hline \multicolumn{2}{|l|}{ Place of death } \\
\hline ED & $157(67.1)$ \\
\hline Intensive care unit & $72(30.8)$ \\
\hline General ward & $4(1.7)$ \\
\hline Operating room & $1(0.4)$ \\
\hline \multicolumn{2}{|l|}{ PS of TRISS } \\
\hline PS $<25 \%$ & $82(35.0)$ \\
\hline $25 \% \leq \mathrm{PS} \leq 75 \%$ & $81(34.6)$ \\
\hline PS $>75 \%$ & $71(30.3)$ \\
\hline \multicolumn{2}{|l|}{ Day of injury } \\
\hline Non-holiday & $168(71.8)$ \\
\hline Holiday & $66(28.2)$ \\
\hline Injury severity score & $24.8 \pm 13.8$ \\
\hline Time from accident to ED arrival (hr) & $1.00(0.42-2.84)$ \\
\hline Time from ED arrival to death (hr) & $2.74(1.11-19.87)$ \\
\hline
\end{tabular}

Values are presented as number (\%), mean \pm standard deviation, or median (interquartile range).

PS, probability of survival; TRISS, Trauma and Injury Severity Score; ED, emergency department.
Table 2. Comparison of general characteristics between preventable and non-preventable trauma deaths

\begin{tabular}{|c|c|c|c|}
\hline Variable & $\begin{array}{l}\text { Preventable } \\
\quad(n=59)\end{array}$ & $\begin{array}{l}\text { Non-preventable } \\
\quad(n=175)\end{array}$ & P-value \\
\hline Age (yr) & $56.8 \pm 20.8$ & $57.5 \pm 18.4$ & 0.810 \\
\hline Sex & & & 0.192 \\
\hline Male & 39 (22.9) & $131(77.1)$ & \\
\hline Female & $20(31.3)$ & $44(68.8)$ & \\
\hline Injury severity score & & & 0.397 \\
\hline$\geq 16$ & $49(24.3)$ & $153(75.7)$ & \\
\hline$<16$ & $10(31.3)$ & $22(68.8)$ & \\
\hline Mode of arrival & & & 0.022 \\
\hline Transferred & 33 (32.7) & $68(67.3)$ & \\
\hline Direct & $26(19.5)$ & $107(80.5)$ & \\
\hline Time from accident to ED arrival & $3.1 \pm 6.1$ & $9.6 \pm 52.2$ & 0.336 \\
\hline Time from ED arrival to death & $23.8 \pm 39.1$ & $21.7 \pm 40.3$ & 0.725 \\
\hline Day of injury & & & 0.014 \\
\hline Holiday & $24(36.4)$ & $42(63.6)$ & \\
\hline Non-holiday & $35(20.8)$ & $133(79.2)$ & \\
\hline Cause of death & & & $<0.001$ \\
\hline Head injury & $11(9.3)$ & $107(90.7)$ & \\
\hline Non-head injury & $48(41.4)$ & $68(58.6)$ & \\
\hline Time admitted to ED & & & 0.007 \\
\hline Day & $18(16.1)$ & $94(83.9)$ & \\
\hline Night & $27(32.1)$ & $57(67.9)$ & \\
\hline Dawn & $14(36.8)$ & $24(63.2)$ & \\
\hline
\end{tabular}

Values are presented as mean \pm standard deviation or number $(\%)$. $E D$, emergency department.

Table 3. Logistic regression analysis for factors associated with preventability of trauma deaths

\begin{tabular}{|c|c|c|c|c|}
\hline \multirow{2}{*}{$\begin{array}{l}\text { Factor } \\
\text { Mode of arrival }\end{array}$} & \multirow[t]{2}{*}{$\begin{array}{c}\text { Adjusted } \\
\mathrm{OR}^{\mathrm{a})}\end{array}$} & \multicolumn{2}{|c|}{ 95\% confidence interval } & \multirow[t]{2}{*}{ P-value } \\
\hline & & & & \\
\hline Direct & Reference & & & \\
\hline Transfer & 2.649 & 1.333 & 5.265 & 0.005 \\
\hline \multicolumn{5}{|l|}{ Time admitted to ED } \\
\hline Day & Reference & & & \\
\hline Night & 2.246 & 1.065 & 4.737 & 0.033 \\
\hline Dawn & 4.173 & 1.612 & 10.802 & 0.003 \\
\hline \multicolumn{5}{|l|}{ Cause of death } \\
\hline Head injury & Reference & & & \\
\hline Non-head injury & 7.104 & 3.344 & 15.092 & $<0.001$ \\
\hline
\end{tabular}

$\mathrm{OR}$, odds ratio; $\mathrm{ED}$, emergency department.

${ }^{a)}$ Adjusted for all variables used in univariate analysis.

deaths regarding each variable were compared. The mode of arrival, day of injury, cause of death, and time admitted to ED showed statistical significance (Table 2). To analyze the factors associated with PDR, we performed logistic regression analysis based on the variables evaluated in the univariate analyses. PDR was significantly higher in cases of transfers from other hospitals $(\mathrm{OR}$, 2.649; $95 \% \mathrm{Cl}, 1.333$ to $5.265 ; \mathrm{P}=0.005)$, transfers at night $(\mathrm{OR}$, $2.246 ; 95 \% \mathrm{Cl}, 1.065$ to $4.737 ; \mathrm{P}=0.033)$ and dawn $(\mathrm{OR}, 4.173$; 
Table 4. Preventable factors by location and process

\begin{tabular}{lccccc}
\hline & System inadequacy & Inadequate treatment or management & Inadequate diagnosis & Inadequate technique & Others \\
\hline Prehospital & $30(52.6)$ & $13(22.8)$ & $2(3.5)$ & $2(3.5)$ & $10(17.5)$ \\
Interhospital transfer & $3(50)$ & $3(50)$ & $0(0)$ & $0(0)$ & $0(0)$ \\
Emergency department & $1(1.4)$ & $64(90.1)$ & $0(0)$ & $0(8.5)$ & $1(100)$ \\
Operation & $0(0)$ & $0(0)$ & $0(0)$ & $1(11.1)$ & $0(0)$ \\
Intensive care unit & $0(0)$ & $1(66.7)$ & $0(0)$ & $0(0)$ & $0(0)$ \\
General ward & $0(0)$ & $1(100)$ & & & $0(0)$ \\
\hline
\end{tabular}

Values are presented as number (\%).

95\% $\mathrm{Cl}, 1.612$ to $10.802 ; \mathrm{P}=0.003)$, and non-head injuries (OR, 7.104; $95 \% \mathrm{Cl}, 3.344$ to $5.092 ; \mathrm{P}<0.001$ ) (Table 3).

\section{Preventable factors during care}

A total of 145 preventable factors were discovered in the 59 preventable deaths, equating to roughly 2.46 preventable factors per death. When factors were categorized by location, ED was the most common location, with 71 preventable factors (49\%), followed by 57 prehospital preventable factors (39.3\%), 9 ICU preventable factors (6.2\%), and 6 during interhospital transfers (4.1\%). Only 1 preventable factor was found in both the operating room and general ward, respectively.

When preventable factors were classified by process, 111 (76.6\%) were process-related, while 34 (23.4\%) were structure-related. Among the process-related factors, inadequacy in treatment or management caused the most preventable factors.

Of 57 prehospital preventable factors, 30 related to structure; of these, 11 concerned transfers. Inadequate treatment or management followed with 13 cases, 9 of which were related to the resuscitation procedures. Six interhospital preventable factors included 3 structure-related preventable factors such as delayed transfer leading to delayed treatment and transfer to a hospital that was inappropriate for post-cardiac arrest care. The other 3 preventable factors were related to inadequate management such as improper airway management and fluid resuscitation. Of the 71 preventable factors related to the ED, 1 was related to structure, while 64 were caused by inadequate treatment. Among these, 35 were connected to resuscitation-related preventable factors, and 17 were linked to setbacks or even errors related to transfusion. Moreover, 4 of 6 preventable factors related to the ICU and inadequate treatment were also associated with resuscitation; however, most of these were linked to breathing or ventilation. Only 1 preventable factor occurred in the general ward; this was also related to resuscitation (Table 4).

\section{Inter-investigator agreement}

Kappa values to evaluate agreement between two investigators were 0.63 in the overall preventable death phase and 0.62 in inhospital preventable death phase.

\section{DISCUSSION}

PDR in Daegu was 25.2\%, and patients who were transferred from other hospitals, transferred at night or dawn, and had non-head injuries had a higher PDR. In prehospital phase, the majority of preventable factors related to structure, while in the in-hospital phase, process-related factors were more common.

PDR in this study was lower than that found in previous Koreabased studies but higher than the 2.5\% to $15.2 \%$ PDR found in foreign studies. ${ }^{12-14}$ While various reasons might explain the discrepancy between this study and those foreign studies, the different methodologies used make a simple parallel comparison difficult. First, the inclusion criteria varied in each study. Saltzherr et al. $^{15}$ pointed this out and noted that their study did not consider prehospital causes. Jung et al. ${ }^{5}$ considered that the differences in the yielded preventability were caused by whether the vital sign data were gathered in the acute phase of the accident or at the time of hospital arrival. Second, geographic and demographic variations, along with different systems for trauma response, end in varying results. In the study by Chiara et al., ${ }^{16}$ performed in Milan, Italy, which has half the population of Daegu but contains 5 level I trauma centers and 8 level II or III trauma centers, the reported PDR was 43\%. However, a British study showed that, even in the same geographic region, inequalities in the trauma response system between medical facilities caused PDR to vary from $0 \%$ to $26 \% .^{8}$ Third, when a study uses a panel review method, investigation by specialists may be affected by each investigator's subjective judgment, and organization of the investigative group may also affect the outcome. Lastly, in Korea, autopsies for post-mortem examinations are not commonly performed due to traditional local customs and lack of legislative policies. Insufficient data from post-mortem examinations may lead investigators to rely on other data sources, such as computed tomography images, to assess the severity of trauma. If autop- 
sies were performed to explore the causes of NP, PDR could potentially decrease. Even after considering these points, Korean studies still tend to show higher PDR than overseas studies. Considering that PDR is a tool to evaluate trauma management systems, such a trend among Korean studies may reflect the inferiority of Korea's trauma response system. However, the present results have improved compared to those of previous studies, which may indicate that the Korean trauma management system has improved over the years. To date, only limited baseline studies exist to portray the characteristics of Daegu's geographic area. Based on the findings in this study we propose to establish a system and methodology that are specific to Daegu and that are able to continuously identify and report on the trauma death rate.

Among the methods used to assess PDR, this study used a panel of specialists, a method that is widely used elsewhere. In studies performed outside of Korea, the investigative panels or committees comprised experts of various occupations such as trauma surgeons, general surgeons, and emergency medical technicians..$^{14,17}$ On the contrary, most Korean studies were investigated only by emergency physicians or a few clinical specialists. Thus, this study is meaningful because other specialists, including trauma surgeons, helped study the domestic PDR. In reaching the final decision by majority vs. unanimity, discrepancies between the preliminary investigators may occur. Mackenzie et al. ${ }^{18}$ showed that the consistency in non-head traumatic injuries was as low as 36\%. To enhance a study's credibility, efforts are needed to reduce such discrepancies, and discussion by a variety of experts can improve the consistency. ${ }^{19}$ Moreover, the consistency is known to increase when the panel comprises only physicians with a single specialty. In this study, the degree of consistency in the overall phase was 0.63 by Kappa values, which was higher than the value in the study by Mackenzie et al. ${ }^{18}$ but lower compared to the values of other Korean studies. ${ }^{7,20}$

This study revealed some problems with the trauma response system in Daegu. First, transferred patients from other hospitals showed a relatively high PDR. In a previous study, ${ }^{21}$ direct visits to the trauma center reduced the length of hospital stay, length of ICU admission, and mortality rate, compared to transfers from other hospitals. Because this study did not review transferred patients' previous hospital records, we were not able to analyze pretransfer trauma care in detail. Nevertheless, increased education about trauma care, sufficient personnel, and consistent investment in the trauma response system may be appropriate given that interhospital transfers in Korea are done mainly by hospital ambulances or private transfer services.

Second, concerning the time of admission to the ED, PDR was higher at dawn than during the day. This finding can be explained by the fact that, while the ED has more patients in the daytime, more medical personnel are present to compensate for the higher burden. On the contrary, although the ED has fewer patients at dawn, only minimum personnel are on duty, and medical activities, including special treatments and procedures, are more limited. In many hospitals, trauma-related specialists are not on call but receive notification from residents and general practitioners. This process is very likely to produce delays or insufficiencies in decision-making and specialized treatment during the dawn. If regional trauma centers had specialists on call 24 hours a day, such weaknesses would be largely resolved.

Finally, non-head injury patients showed a high PDR in this study. In our study, 22 patients classified as preventable deaths had hemorrhage as the cause of death. Of those patients, inadequate fluid resuscitations, delays in diagnosing hemorrhage, and delays in transfusion due to in-hospital processes occurred in 14 cases. Transfusion is different from other fluid or drug administration because it requires $A B O$ typing, antibody screening, and cross-matching. This process makes prompt transfusion in the early trauma stage very difficult. To solve this issue, some have suggested transfusing universal donor blood ( 0 in $A B O$ typing and $\mathrm{Rh}[-]$ ) in early trauma, during the full screening for transfusion. ${ }^{22}$ Many studies have shown that the mortality did not increase significantly using this approach. ${ }^{23,24}$ Alternatively, transfusing 0 but Rh (+) blood or administering hemoglobin-based oxygen carriers as blood substitutes have also been proposed. ${ }^{25-27}$ In this study, PDR of head-injured patients as the cause of death was $9.3 \%$, which is considerably lower than the corresponding rate of $18.5 \%$ in the study by Kim et al. ${ }^{28}$ The majority of identified preventable factors were due to structural preventable factors in the prehospital stage (4 of 6 cases) and inadequate treatment or management in the ED (13 of 16). Preventable factors in the ED were associated mainly with ED monitoring or dispatch. To overcome these preventable factors, implementation of the Brain Trauma Foundation guidelines into treatment could improve the survival outcome among patients with brain injuries. ${ }^{29}$

In the prehospital phase, $52.6 \%$ of preventable factors were related to structure. Such preventable factors included the frequent selection of inadequate hospitals, including hospitals unsuited for treating severe trauma patients, facilities incapable of surgical procedures, or remote hospitals with long transfer distances. The standard protocols for EMS providers include a hospital-selection guideline for 119 dispatchers (Korea's emergency number). However, according to one study, ${ }_{1}^{30}$ with respect to transferring trauma patients in Daegu, the rate of violating the guideline was over $50 \%$. The rate of guideline violation combined with bypassing transfer was $46.2 \%$. Improving the proficiency of 
EMS personnel through constant education and evaluation may reduce such preventable factors. Furthermore, 5 emergency medical centers in Daegu are concentrated in a certain region, and some specialized trauma centers are under operation, so EMS dispatchers might select hospitals based on subjective judgment. Therefore, we suggest creating guidelines for hospital transfer that reflect the special characteristics of medical resources in Daegu.

In the in-hospital phase, most preventable factors developed in the ED and usually related to inadequate treatment. Although 5 emergency medical centers participating in this study treated severe trauma patients in Daegu, with the exception of 1 regional emergency medical center, 4 local emergency medical centers did not have in-hospital trauma care protocols. Such absence of standardized protocols may lead to inadequate treatment.

This study has some limitations. First, upon determining preventable trauma deaths, the autopsy findings were not taken into account. Many former Korean studies have also pointed out this issue; however, this study was a baseline study to ensure basic data for a local trauma system. If future studies are performed using the same target patients and methodology, we may be able to compare the preventability. Second, when patients were transferred from other hospitals, we could not use the medical records from the transferring hospitals. If this information could be analyzed in future studies, weak points of not only the treatment process from the transferring hospital but also the emergency medical delivery system of severe trauma patients could be reviewed in detail. For instance, if patient transfer to the final hospital were delayed, we may be able to identify whether the reason for delay was that 1) the transfer decision was hastened because of an unnecessary diagnostic procedure or delayed diagnosis, 2) essential primary care and stabilization of vital signs were needed after early assessment, or 3) the diagnosis was made early but selecting the transfer hospital was difficult, therefore requiring a long time to confirm the facility's capacity.

In summary, the PDR in Daegu was 25.2\%. This study is meaningful because it was the first to investigate the PDR in the Daegu area. Efforts to mediate the preventable factors revealed in this study and continuous reviews to calculate and track the PDR are needed to evaluate the local trauma system. This study may also be used as a cornerstone in establishing a trauma management system specialized for Daegu.

\section{CONFLICT OF INTEREST}

No potential conflict of interest relevant to this article was reported.

\section{REFERENCES}

1. Korean Statistical Information Service. Deaths, death rates, age-standardized death rates by cause (50 item) and sex: si, gun, and gu [Internet]. Daejeon: Statistics Korea; 2014 [cited 2015 Aug 31]. Available from: http://kosis.kr/statisticsList/ statisticsList_01 List.jsp?vwcd = MT_ZIITLECtparentld = D.

2. Rutstein DD, Berenberg W, Chalmers TC, Child CG 3rd, Fishman AP, Perrin EB. Measuring the quality of medical care: a clinical method. N Engl J Med 1976;294:582-8.

3. Settervall CH, Domingues Cde A, Sousa RM, Nogueira Lde S. Preventable trauma deaths. Rev Saude Publica 2012;46:36775.

4. Chiara O, Cimbanassi S, Pitidis A, Vesconi S. Preventable trauma deaths: from panel review to population based-studies. World J Emerg Surg 2006;1:12.

5. Jung KY, Kim JS, Kim Y. Problems in trauma care and preventable deaths. J Korean Soc Emerg Med 2001;12:45-56.

6. Kim Y, Jung KY, Cho KH, et al. Preventable trauma deaths rates and management errors in emergency medical system in Korea. J Korean Soc Emerg Med 2006;17:385-94.

7. Kim H, Jung KY, Kim SP, et al. Changes in preventable death rates and traumatic care systems in Korea. J Korean Soc Emerg Med 2012;23:189-97.

8. Gorman DF, Teanby DN, Sinha MP, Wotherspoon J, Boot DA, Molokhia A. Preventable deaths among major trauma patients in Mersey Region, North Wales and the Isle of Man. Injury 1996;27:189-92.

9. Daegu Statistical Information. Daegu statistical information [Internet]. Daegu: Daegu Statistical Information; 2012 [cited 2015 Aug 31]. Available from: http://statistics.daegu.go.kr/.

10. National Emergency Medical Center. 2012 Emergency medical service statics annual report. Seoul: National Emergency Medical Center; 2013.

11. Kelly CA, Upex A, Bateman DN. Comparison of consciousness level assessment in the poisoned patient using the alert/verbal/painful/unresponsive scale and the Glasgow Coma Scale. Ann Emerg Med 2004;44:108-13.

12. Teixeira $P G$, Inaba $K$, Hadjizacharia $P$, et al. Preventable or potentially preventable mortality at a mature trauma center. J Trauma 2007;63:1338-46.

13. Kleber C, Giesecke MT, Tsokos M, Haas NP, Buschmann CT. Trauma-related preventable deaths in Berlin 2010: need to change prehospital management strategies and trauma management education. World J Surg 2013;37:1154-61.

14. Sanddal TL, Esposito TJ, Whitney JR, et al. Analysis of preventable trauma deaths and opportunities for trauma care impro- 
vement in utah. J Trauma 2011;70:970-7.

15. Saltzherr TP, Wendt KW, Nieboer $P$, et al. Preventability of trauma deaths in a Dutch level-1 trauma centre. Injury 2011;42: 870-3.

16. Chiara O, Scott JD, Cimbanassi $S$, et al. Trauma deaths in an Italian urban area: an audit of pre-hospital and in-hospital trauma care. Injury 2002;33:553-62.

17. McDermott FI, Cordner SM, Cooper DJ, Winship VC; Consultative Committee on Road Traffic Fatalities in Victoria. Management deficiencies and death preventability of road traffic fatalities before and after a new trauma care system in Victoria, Australia. J Trauma 2007;63:331-8.

18. MacKenzie EJ, Steinwachs DM, Bone LR, Floccare DJ, Ramzy Al. Inter-rater reliability of preventable death judgments. The Preventable Death Study Group. J Trauma 1992;33:292-302.

19. MacKenzie EJ. Review of evidence regarding trauma system effectiveness resulting from panel studies. J Trauma 1999;47(3 Suppl):S34-41.

20. Kang JH, Park KH, Kim WJ, et al. Problems of trauma care and rate of preventable trauma death in Jeju, South Korea. J Korean Soc Emerg Med 2011;22:438-45.

21. Young JS, Bassam D, Cephas GA, Brady WJ, Butler K, Pomphrey M. Interhospital versus direct scene transfer of major trauma patients in a rural trauma system. Am Surg 1998;64:88-91.

22. Boisen ML, Collins RA, Yazer MH, Waters JH. Pretransfusion testing and transfusion of uncrossmatched erythrocytes. An- esthesiology 2015;122:191-5.

23. Schwab CW, Shayne JP, Turner J. Immediate trauma resuscitation with type 0 uncrossmatched blood: a two-year prospective experience. J Trauma 1986;26:897-902.

24. Inaba K, Teixeira PG, Shulman I, et al. The impact of uncrossmatched blood transfusion on the need for massive transfusion and mortality: analysis of 5,166 uncross-matched units. J Trauma 2008;65:1222-6.

25. Dutton RP, Shih D, Edelman BB, Hess J, Scalea TM. Safety of uncrossmatched type- 0 red cells for resuscitation from hemorrhagic shock. J Trauma 2005;59:1445-9.

26. Sarkar S. Artificial blood. Indian J Crit Care Med 2008;12:140-4.

27. Kresie L. Artificial blood: an update on current red cell and platelet substitutes. Proc (Bayl Univ Med Cent) 2001;14:158-61.

28. Kim SC, Song KJ, Shin SD, Lee SC, Park JO, Holmes JF. Preventable deaths in patients with traumatic brain injury. Clin Exp Emerg Med 2015;2:51-8.

29. Brain Trauma Foundation; American Association of Neurological Surgeons; Congress of Neurological Surgeons. Guidelines for the management of severe traumatic brain injury. J Neurotrauma 2007;24 Suppl 1:S1-106.

30. Choi YH, Ahn KO, Shin SD, et al. Compliance of a bypassing hospital trauma protocol using the field triage decision scheme between metropolitan vs non-metropolitan emergency medical services. J Korean Soc Emerg Med 2015;26:138-48. 